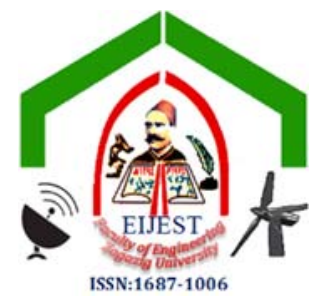

The Egyptian International Journal of Engineering Sciences and Technology

Vol. 19 No. 2 (2016-Special Issue) 297-304

\title{
A Generic Mathematical Model to Optimize the Integrted Production, Inventory and Distribution Decisions in Supply Chains
}

\author{
Noha A. Mostafa* and Amr B. Eltawil \\ Department of Industrial Engineering and Systems Management, Egypt-Japan University of Science and Technology, New Borg El Arab, 21934, \\ Alexandria, Egypt
}

\begin{tabular}{|c|c|}
\hline A R T I CLE I N F O & A B S T RACT \\
\hline $\begin{array}{l}\text { Article history: } \\
\text { Received 17 May } 2016 \\
\text { Accepted 19 June } 2016 \\
\text { Available online 1 July } 2014 \\
\end{array}$ & $\begin{array}{l}\text { Integration of decisions in supply chain management has received increasing } \\
\text { consideration recently. The Production-Inventory-Distribution-Routing Problem } \\
\text { (PIDRP) is a recent and complex problem that integrates decisions on lot-sizing, }\end{array}$ \\
\hline $\begin{array}{l}\text { Keywords: } \\
\text { Supply chain management; } \\
\text { Production planning; } \\
\text { Inventory; } \\
\text { Distribution; } \\
\text { Vehicle routing. }\end{array}$ & $\begin{array}{l}\text { inventory management, distribution planning, and vehicle routing problems. In this } \\
\text { paper, a generic problem description is given and a Mixed-Integer Programming } \\
\text { model (MIP) is proposed to solve the PIDRP, the objective is to minimize the total } \\
\text { cost of the combined functions while satisfying the required service levels. The } \\
\text { proposed model contributes to the existing literature since it deals with multiple } \\
\text { products, split deliveries, a heterogeneous fleet of vehicles, and puts a limit on the } \\
\text { duration of the route performed by each vehicle. The proposed model was } \\
\text { successfully validated and tested by using small-sized instances from literature. Also, } \\
\text { a sensitivity analysis was performed to investigate the effect of estimated parameters } \\
\text { on the model results. }\end{array}$ \\
\hline
\end{tabular}

\section{Introduction}

Supply Chain Management (SCM) is a set of approaches utilized to integrate suppliers, manufacturing, warehouses, and stores so that products are produced and distributed at the right quantities, to the right location, at the right time, in order to minimize system-wide costs while satisfying service level requirements [1]. The main SCM functions are location and network design, marketing, purchasing, production, inventory, distribution, and vehicle routing [2].

The integration between different SCM functions has become a key factor of success recently. Focusing on cost reduction in one area of the supply chain often leads to higher costs in other areas [3]. In general, modelling more comprehensive problems creates the opportunity for more savings [4]. Several studies were made on the partial integration of SCM functions resulting into some important and well-known problems, such as the Inventory Routing Problem (IRP), the Location Routing Problem (LRP), and the Production-Distribution Problem (PDP). The most recent integrated SCM problem is the ProductionInventory-Distribution-Routing Problem (PIDRP).

The PIDRP is a complex and large problem that integrates tactical and operational decisions on production lot-sizing, inventory management, distribution policy and quantities, and vehicle routing, with the objective of minimizing the total cost. The PIDRP can be seen as an extension of the Inventory Routing Problem (IRP), by including production decisions which are not considered in the IRP [5].

* Corresponding author. Tel.: +20-100-4242-672.

E-mail address: noha.ali@ejust.edu.eg 
Since 2006, the research on the PIDRP gained the research interest to find good modelling and solution approaches. However, the research in this area is relatively limited, with many potential research points. For a recent review on the works addressing the PIDRP, refer to [2, 5].

Hence, it can be seen that the PIDRP is the most comprehensive SCM problem so far, and it, also, represents a recent and promising research direction. The main contribution of this work is to provide a novel and generic mathematical formulation for the problem with computational results for data instances from literature and sensitivity analysis.

This problem can be seen in industry in applications such as the production and distribution of dairy and bakery products. The products of such industries are characterised by short shelf life and special inventory and distribution requirements. One important aspect of such distribution networks is the presence of a large number of stops with small drops or deliveries at each stop. Another aspect is the fact that, the delivery trucks may be dropping and collecting perished items from the visited location to return them back to the facility. However, the issue of reverse logistics is not considered in this paper.

The paper is organized as follows; in section 2, a literature review for the PIDRP is given. The proposed MIP model for the PIDRP is presented in section 3. Section 4 gives the computational results and sensitivity analysis, and Section 5 gives the conclusions.

\section{Literature Review}

The research on integrating decisions on different SCM functions started from nearly thirty years ago, and increased considerably during the past decade. The research on the PIDRP was motivated by the work of Chandra and Fisher [6] who suggested that coordinating the decisions on these different functions into a single model instead of solving them separately can achieve a cost reduction between $3 \%$ and $20 \%$. Although these results were promising, the research on this integrated problem was relatively slow, that was due to the complexity of the problem and limitations of the computational abilities.

In 2006, the work in [7] regained the interest in the PIDRP, a two-phase approach was used to solve the problem of multiple plants that produce a single product and distribute it to multiple distribution centres with a fleet of heterogeneous vehicles. In phase I, a mathematical model was used to optimize decision variables of lot-sizing, inventory, and distribution with restricting the routing constraints to direct shipments only. Phase II applies a load consolidation heuristic to solve an associated consolidation problem.

Due to the complexity of the problems, researchers started to resort to using metaheuristics. In [8], a greedy randomized adaptive search procedure (GRASP) was used to tackle simultaneously production and routing decisions, and then it was improved by a reactive mechanism or by a path relinking process for better results. In [9] the same authors used a greedy heuristic with a saving algorithm for the PIDRP. Then, a local search procedure based on 3-opt moves, insertion, and swap heuristics was used to improve the solution. Memetic algorithm with population management was used in [10], this algorithm could deal simultaneously with production and distribution decisions.

In $[11,12,13]$ Bard and Nanaukul presented three important works on the PIDRP for single production plant, single product, multiple customers and a fleet of homogeneous vehicles. In [11], an allocation model was used to find good starting feasible points for a reactive Tabu search procedure with path relinking was used to solve the PIRDP. In [12], a column generation scheme was used to determine delivery quantities for each customer in each time period, and then a two-step heuristic was proposed to solve the IRP component and find the actual routes by using Tabu search. In [13], a branch-and-price-based scheme was proposed for solving the PIDRP; several methods were introduced to deal with symmetry during branching.

A mathematical model for the multi-commodity PIDRP was proposed in [14]; the model considers many issues such as production capacity limitation, inventory accumulation, storage limits, and vehicle routing. Results showed that implementing the model may result in reduction in holding costs and energy consumption of refrigerated storage.

In 2011, two notable works were presented; in [15] different inventory policies were considered for solving a single retailer, single vehicle PIDRP. A novel Hybrid heuristic was used with very good results compared to the exact procedure. In [16], two Tabu search variants for the PIDRP were presented; the first variant involved a construction phase and a short-term memory algorithm, and the second incorporates a longer term memory used to integrate a path relinking procedure to the first variant. The results showed that the proposed procedures can be successfully applied to the PIDRP and can be extended to other planning problems with a discrete time horizon. 
A Decision Support System (DSS) was proposed in [17] by combining a database management system and a mathematical model to solve a PIDRP for multiple plants and multiple products, the results showed that the proposed DSS works interactively with the optimization model providing flexibility in real-time decisions.

Recently, in [18], a two-phase iterative heuristic was proposed with a maximum level inventory policy based on the work in [15]; the first phase was for lotsizing with vehicle capacity consideration, the second phase was for routing decisions. Two diversification mechanisms were used to prevent quick convergence to local optimum. Adulyasak et al. in [3, 19] presented an Adaptive Large Neighbourhood Search (ALNS) procedure to solve the PIDRP. The results showed that this algorithm outperforms existing heuristics for the PIDRP with respect to quality and computational time.

It was seen from the survey on the PIDRP, that problem is gaining increased consideration in recent years. However, the available models and approaches are, mostly, limited to less complex cases. The literature on the PIDRP is relatively few, providing good opportunity for further research.

\section{MIP Formulation of the PIDRP}

\subsection{Assumptions of the PIDRP model}

The following assumptions are used for the MIP model proposed for the PIDRP:

- A single capacitated production plant.

- Multiple types of products, to be delivered to multiple geographically dispersed customers.

- Products have different setup costs, unit operational costs, and production capacities.

- Deterministic demand.

- Inventory can be kept in both the plant and customer facilities with limited amount.

- Delivery is performed by a fleet of capacitated heterogeneous vehicles.

- Vehicles can be loaded with different types of products.

- The route duration of any vehicle cannot exceed a predetermined planning period's duration.

- All vehicles should return to the plant at the end of the planning period.

- Shortages are not allowed.

- Splits deliveries are allowed

\subsection{Notation of the PIDRP model}

Indices

$t=1,2, \ldots, T \quad$ Set of planning periods

$i, j=0$, Set of facilities, where 0

$1, \ldots, N \quad$ corresponds to production plant, and other nodes represent customers, $i \neq j$

$k=1,2, . ., \quad$ Set of products

K

$v=1,2, . ., \quad$ Set of vehicles

V

\section{Parameters}

$s_{k} \quad$ Production setup cost of product $k$

$o_{k} \quad$ Unit production cost of product $k$

$c_{k} \quad$ Production capacity of product $k$ per each period

$C$ Total production capacity of the production plant

$h_{0 k} \quad$ Per unit inventory holding cost of product $k$ at the production plant

$h_{i k} \quad$ Per unit inventory holding cost of product $k$ at customer $i$

$M_{i k} \quad$ Maximum allowed inventory level of product $k$ at facility $i$

$d_{i k t} \quad$ Demand of product $k$ at customer $i$ in period $t$

$q_{v} \quad$ Capacity of vehicle $v$

$f_{v} \quad$ Fixed transportation cost of using vehicle $v$

$r_{i j v} \quad$ Routing cost for traveling from node $i$ to node $j$ using vehicle $v$

$l_{i j v} \quad$ Expected duration to travel from node $i$ to node $j$ using vehicle $v$

$L \quad$ Fixed length of each planning period

$u_{k} \quad$ Specific loading characteristic of product $k$

$\gamma \quad$ A large number

\section{Decision variables}

$Q_{k t} \quad$ Production quantity of product $k$ in period $t$

$P_{k t} \quad$ Binary; 1 if product $k$ is produced in period $t, 0$ otherwise

$I_{0 k t} \quad$ Inventory level of product $k$ at the production plant at the end of period $t$

$I_{i k t} \quad$ Inventory level of product $k$ at customer $i$ at the end of period $t, i \neq 0$

$X_{i k t v} \quad$ Amount of product $k$ delivered to customer $i$ by vehicle $v$ in period $t, \mathrm{i} \neq 0$

$W_{i t} \quad$ Binary; 1 if customer $i$ is visited in period $t, 0$ otherwise

$Y_{i j k t v} \quad$ Amount of product $k$ delivered between node $i$ to node $j$ by vehicle $v$ in period $t$

$Z_{i j v} \quad$ Binary; 1 if vehicle $v$ travels between node $i$ to node $j$ in period $t, 0$ otherwise 


\subsection{Mathematical Formulation}

The formulations proposed in $[13,16,19]$ were extended to propose a generic formulation of the PIDRP is as follows:

Minimize

$$
\begin{aligned}
\sum_{t \in T}\left[\sum _ { k \in K } \left[s_{k} P_{k t}+\right.\right. & \left.o_{k} Q_{k t}+h_{0 k} I_{0 k t}+\sum_{i \in N \backslash\{0\}} h_{i k} I_{i k t}\right] \\
& +\sum_{v \in V}\left[\sum_{i \in N \backslash\{0\}} f_{v} Z_{0 i t v}\right. \\
& \left.\left.+\sum_{i \in N} \sum_{j \in N \backslash\{i\}} r_{i j v} Z_{i j t v}\right]\right]
\end{aligned}
$$

Subject to

$$
\begin{array}{lr}
Q_{k t} \leq \gamma P_{k t} & \forall k \in K, \forall t \in T \\
Q_{k t} \leq c_{k} P_{k t} & \forall k \in K, \forall t \in T \\
\sum_{k \in K} Q_{k t} \leq C & \forall t \in T \\
I_{0 k t}=Q_{k t}+I_{0 k t-1}-\sum_{i \in N \backslash\{0\}} \sum_{v} X_{i k t v} & \forall k \in K, \forall t \in T \\
I_{i k t}=\sum_{v} X_{i k t v}+I_{i k t-1}-d_{i k t} & \forall i \in N \backslash\{0\}, \forall k \in K, \forall t \in T \\
I_{i k t} \leq M_{i k} & \forall i \in N, \forall k \in K, \forall t \in T \\
\sum_{k \in K} Y_{i j k t v} \leq q_{v} Z_{i j t v} & \\
\forall i \in N, j \in N \backslash\{i\}, \forall v \in V, \forall t \in T & \forall v \in V, \forall t \in T
\end{array}
$$$$
\forall i \in N, \forall k \in K, \forall t \in T
$$$$
\sum_{i \in N \backslash\{0\}} \sum_{j \in N \backslash\{i\}} l_{i j v} Z_{i j t v} \leq L
$$

$\forall v \in V, \forall t \in T$

$$
\sum_{i \in N \backslash\{0\}} \sum_{v \in V} Y_{\text {oiktv }}=\sum_{i \in N \backslash\{0\}} \sum_{v \in V} X_{i k t v}
$$$$
\forall k \in K, \forall t \in T
$$

$$
\begin{aligned}
& \sum_{j \in N \backslash\{i\}} Y_{j i k t v}-\sum_{\substack{j \in N \backslash\{i\} \\
\forall i \in N \backslash\{0\}, \forall k \in K, \forall v \in V, \forall t \in T}} Y_{i j k t v}=X_{i k t v} \\
& \sum_{i \in N} \sum_{j \in N \backslash\{i\}} Z_{i j t v} \leq 1
\end{aligned}
$$

$\forall v \in V, \forall t \in T$

$$
\sum_{j \in N \backslash\{i\}} Z_{j i t v}-\sum_{j \in N \backslash\{i\}} Z_{i j t v}=0
$$

$\sum_{j \in N \backslash\{i\}} Z_{j i t v}+\sum_{j \in N \backslash\{i\}} Z_{i j t v}=2 W_{i t}$

$\forall i \in N, \forall v \in V, \forall t \in T$

$$
\begin{array}{lr}
Q_{k t} \geq 0 & \forall k \in K, \forall t \in T \\
I_{0 k t} \geq 0 & \forall k \in K, \forall t \in T \\
I_{i k t} \geq 0 & \forall i \in N \backslash\{0\}, \forall k \in K, \forall t \in T \\
X_{i k t v} \geq 0 & \forall i \in N \backslash\{0\}, \forall k \in K, \forall v \in V, \forall t \in T \\
Y_{i j k t v} \geq 0 & \forall i \in N \backslash\{j\}, \forall j \in N \backslash\{i\}, \forall \in K, \\
& \forall v \in V, \forall t \in T \\
P_{k t} \in\{0,1\} & \forall k \in K, \forall t \in T \\
W_{i t} \in\{0,1\} & \forall i \in N \backslash\{0\}, \forall t \in T \\
Z_{i j t v} \in\{0,1\} & \forall i \in N \backslash\{j\}, \forall j \in N \backslash\{i\}, \\
\forall v \in V, \forall t \in T
\end{array}
$$

The objective function (1) minimizes the summation of three types of costs; total production cost (setup and operational costs), inventory holding costs at the production plant and customer sites, and finally the transportation cost (fixed and routing costs). The constraints (2-24) represent the assumptions and limitations for the production, inventory, distribution, and vehicle routing.

\section{Computational Results and Sensitivity Analysis}

In this section, the proposed model, presented in section 3, was validated and tested for small-sized data instances. The model was implemented by using LINGO 15.0 optimization software and tested on a 2.2 $\mathrm{GHz}$ Core i3 with 4 GB RAM under Windows 7 environment.

To validate the model, it was reduced to solve eight data instances from literature [20]. In [20] a Particle 
Swarm Optimization (PSO) algorithm was proposed to solve the problem for a single product, a homogeneous fleet of vehicles.

Table 1 provides the instance size, the objective function values for the PSO and the proposed MIP model, respectively, the CPU time in seconds for the MIP model, and the gap between the two methods.

Table 1. Validation and improvement by the proposed model

\begin{tabular}{|c|c|c|c|c|c|}
\hline $\mathbf{i}$ & $\begin{array}{c}\text { Size } \\
(\mathbf{N} \times \mathbf{T} \times \mathbf{V})\end{array}$ & OBJ $\mathbf{P S O}$ & $\mathbf{O B J}_{\mathbf{M I P}}$ & $\mathbf{C P \mathbf { U } _ { \mathbf { M I } }}$ & $\mathbf{G a p}$ \\
\hline 1 & $4 \times 5 \times 2$ & 38,369 & 37,579 & 11.1 & $2.06 \%$ \\
2 & $4 \times 10 \times 2$ & 76,739 & 72,108 & 20.0 & $6.03 \%$ \\
3 & $6 \times 5 \times 3$ & 57,554 & 52,553 & 24.3 & $8.69 \%$ \\
4 & $6 \times 10 \times 3$ & 115,108 & 103,046 & 66.5 & $10.5 \%$ \\
5 & $8 \times 5 \times 4$ & 76,739 & 74,290 & 45.4 & $3.19 \%$ \\
6 & $8 \times 10 \times 4$ & 153,478 & 145,700 & 110.2 & $5.07 \%$ \\
7 & $10 \times 5 \times 5$ & 95,924 & 92,442 & 87.2 & $3.63 \%$ \\
8 & $10 \times 10 \times 5$ & 191,004 & 182,601 & 204.9 & $4.40 \%$ \\
\hline
\end{tabular}

From Table 1, it was found that the proposed MIP model was able to solve all the eight instances to optimality in a short time with results' improvement between $2 \%$ and $10 \%$.

After the proposed model was validated, larger data instances were generated by extending the instances from [20] to add an additional product, and defining a heterogeneous fleet of vehicles with the full sets of constraints. Tables 2 to 4 give the data for the first problem that has a production plant, three customers, five time periods, two vehicles, and two products; then the problem size is $(4 \times 5 \times 2 \times 2)$.

Table 2. Parameters of products and vehicles for the first data

\begin{tabular}{|c|c|c|c|c|c|}
\hline Par & Prod 1 & Prod 2 & Par & V 1 & V 2 \\
\hline $\mathrm{s}_{\mathrm{k}}$ & 809 & 536 & $\mathrm{q}_{\mathrm{v}}$ & 24 & 26 \\
$\mathrm{o}_{\mathrm{k}}$ & 89 & 88 & $\mathrm{f}_{\mathrm{v}}$ & 228 & 239 \\
$\mathrm{c}_{\mathrm{k}}$ & 54 & 51 & & & \\
$\mathrm{u}_{\mathrm{k}}$ & 0.2 & 0.1 & & & \\
\hline
\end{tabular}

Table 3. Holding costs for the first data instance for each product in

\begin{tabular}{|c|c|c|}
\hline \multicolumn{3}{|c|}{ each facility } \\
\hline Facility & Product 1 & Product 2 \\
\hline 0 & 10 & 9 \\
1 & 7 & 9 \\
2 & 8 & 9 \\
3 & 9 & 8 \\
\hline
\end{tabular}

Table 4. Demand of the two products for the first data instance

\begin{tabular}{|c|c|c|c|c|c|}
\hline \multirow{2}{*}{$\begin{array}{c}\text { Custome } \\
\mathbf{r}\end{array}$} & \multicolumn{5}{|c|}{ Time Period } \\
\cline { 2 - 6 } & 1 & 2 & 3 & 4 & 5 \\
\hline 1 & 11,11 & 10,13 & 18,14 & 19,19 & 20,14 \\
2 & 20,17 & 15,14 & 14,16 & 11,10 & 17,17 \\
3 & 10,15 & 20,13 & 15,15 & 20,12 & 11,17 \\
\hline
\end{tabular}

The same computations were performed for the other seven instances in Table 1. The results are shown in Table 5. It was found that seven out of the eight problem instances could be solved to optimality in less than four hours. As the problem includes vehicle routing, which is classified as an operational problem, it may be needed to solve the problem on daily basis. Hence, the authors think that a computational time above four hours would be impractical; especially that in the real-life applications it is highly likely to have larger instances.

Table 5. Computational time in seconds for the extended data

\begin{tabular}{|c|c|c|}
\hline \multicolumn{3}{|c|}{ instances } \\
\hline instance & Size $(\mathbf{N} \times \mathbf{T} \times \mathbf{V} \times \mathbf{K})$ & $\mathbf{C P U}$ MIP \\
\hline 1 & $4 \times 5 \times 2 \times 2$ & 948.6 \\
2 & $4 \times 10 \times 2 \times 2$ & 1775.3 \\
3 & $6 \times 5 \times 3 \times 2$ & 3808.1 \\
4 & $6 \times 10 \times 3 \times 2$ & 5667.9 \\
5 & $8 \times 5 \times 4 \times 2$ & 9449.4 \\
6 & $8 \times 10 \times 4 \times 2$ & $12,507.0$ \\
7 & $10 \times 5 \times 5 \times 2$ & $11,052.2$ \\
8 & $10 \times 10 \times 5 \times 2$ & $>14,400$ \\
\hline
\end{tabular}

The large increase in the computational time is caused by the large number of binary variables in the mathematical model and the fact that LINGO uses branch-and-bound methodology for solving such problems. Hence the computational time grows rapidly with the problem size.

Heuristics provide not optimal but faster solutions, many heuristics provide very near solutions to the optimal one. For the large instances, exact methods tend to be very time consuming and impractical for such kind of operational problems, heuristics are more likely to be used. Heuristics and metaheuristics were used by most of the works on the PIDRP. A very recent approach is to combine mathematical programming and metaheuristics to form what is called "matheuristics", this method is very promising and can be used in future works.

\subsection{Sensitivity analysis}

In this section, two sensitivity analyses are performed for two different parameters of the MIP model. From literature, it was found that change production capacity has big influence on the total cost, see $[6,16]$. Also, travel times may be a big source of variation due to traffic jams, road and weather conditions, accidents, etc. [21]. Hence, the sensitivity of the solution should be investigated in response to the changes in these parameters. Parameters $\mathrm{C}$ and $\mathrm{l}_{\mathrm{ijv}}$ are varied in intervals as shown in Table 6 , and then the model was run for 10 runs for 100 variations of each parameter.

Figures 1 and 2 depict the influence of the change in each parameter on the average total cost, average production cost, average inventory cost, average 
transportation cost and average computational time for the eight instances. The response of these different measures relative to each parameter is summarized in Table 7.

Table 6. Intervals for sensitivity analysis for production capacity and travel time

\begin{tabular}{|cc|}
\hline Par & Interval \\
\hline & {$\left[\frac{\left(3 \sum_{i \in N \backslash\{0\}} \sum_{k \in K} \sum_{t \in T} d_{i k t}\right)}{(N-1) \cdot T}, \frac{\left(4 \sum_{i \in N \backslash\{0\}} \sum_{k \in K} \sum_{t \in T} d_{i k t}\right)}{(N-1) . T}\right]$} \\
$1_{\mathrm{ijv}}$ & {$[100,500]$ divided into eight intervals }
\end{tabular}

Table 7. Sensitivity of solution measures to the change in the

\begin{tabular}{|c|c|c|}
\hline & $\begin{array}{c}\text { Production } \\
\text { Capacity }\end{array}$ & Travel time \\
\hline Computational time & + & + \\
\hline Total cost & - & + \\
\hline Production cost & + & + \\
\hline Inventory cost & - & - \\
\hline Transportation cost & + & + \\
\hline
\end{tabular}

\section{Conclusions}

In this work, an integrated problem for supply chain management functions was addressed, that is the production-inventory-distribution-routing problem. It was shown that the research on PIDRP has received increasing research interest during the past decade. However, the research on this problem is relatively few and limited. To contribute to the research on the PIDRP, a mixed-integer programming model was proposed for a generic case and used to solve data instances from literature. Results showed that the proposed model can be used efficiently for small-sized data instances and for solving them to optimality in reasonable time. For larger instances, it was found that the computational time increases drastically; this is due to the combinatorial nature of the problem and the number of binary variables.

Finally, a sensitivity analysis was performed to recognize the influence of changing two key parameters of the model; production capacity and travel time. It was found that increasing the production capacity would decrease the total cost with an affordable increase in the computational time. On the other hand, the increase in travel times would largely increase the total cost with a slight increase in the computational time.

To solve large instances, seeking optimality would be very time consuming and is not guaranteed; that is why heuristics can be used for large instances to get faster solutions with near optimal solutions. Some heuristics and meta heuristics were used previously for the PIDRP. In future work, these methods will be studied and new combinations can be developed to get an efficient approach to solve the problem.

This work can have a significant contribution when used for the perishable products production and distribution industry especially industries with short life time products such as dairy and baked products. One important extension of this work is to include pick-up and delivery in the formulation, allowing for solution of the case of simultaneous forward and reverse logistics.

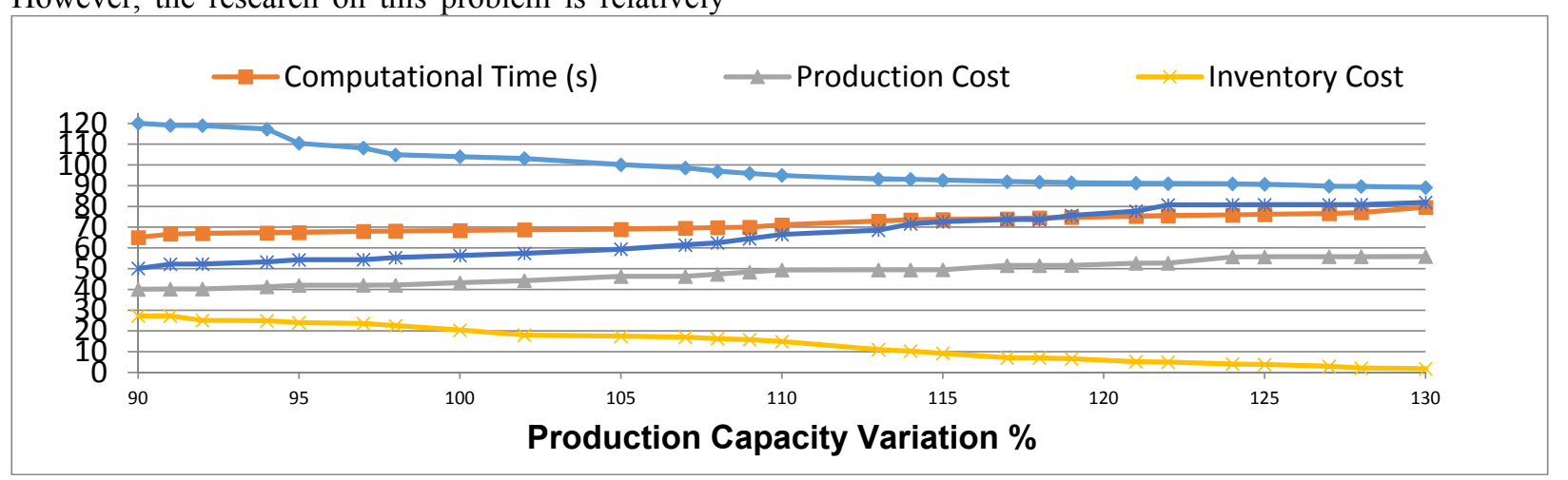

Fig.1. Effect of change in production capacity 


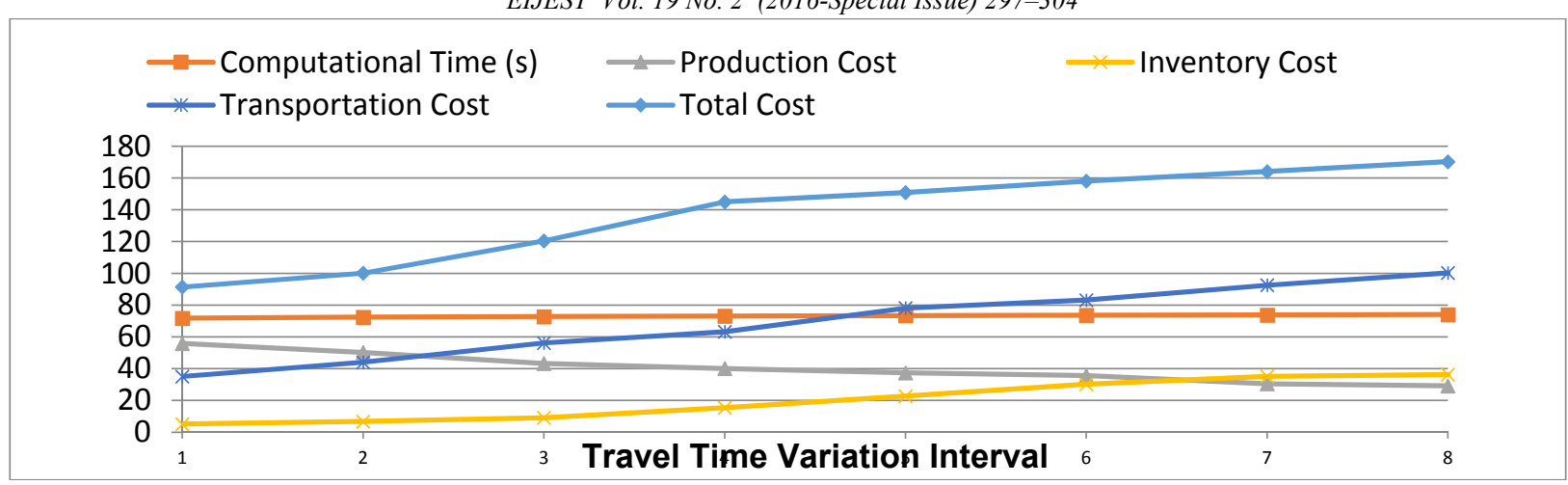

Fig.2. Effect of change in travel time

As many of the products considered for this problem are distributed in refrigerated trucks, another extension can be the consideration of truck emissions in both the objective function as well as in the constraints in a matter related to Pollution routing Problem [22, 23]. In such problem the objective function would not only aim to minimizing cost or total distance but the produced emissions as well.

\section{Acknowledgements}

This research project was supported by the Egyptian ministry of higher education grant and the Japanese International Cooperation Agency (JICA) in the scope of the Egypt-Japan University of Science and Technology (E-JUST).

\section{References}

[1] D. Simchi-Levi, P. Kaminsky, E. Simchi-Levi, "Designing and Managing the Supply Chain: Concepts, Strategies, and Case Studies (3rd edition)", Boston, MA, Irwin/McGraw-Hill, 2008.

[2] N.A. Mostafa and A.B. Eltawil, "The productioninventory-distribution-routing problem: An integrated formulation and solution framework," Proceedings of the Industrial Engineering and Operations Management (IEOM), Dubai, UAE, 2015. Available from:

http://ieeexplore.ieee.org/stamp/stamp.jsp?tp=\&a rnumber $=7093751$ \&isnumber $=7093693$

[3] Y. Adulyasak, J-F. Cordeau, and R. Jans, "Optimization-based adaptive large neighborhood search for the production routing problem," Transportation Science, Vol. 48, No. 1, pp. 20-45, 2014.

[4] L. Bertazzi and M.G. Speranza, "Inventory routing problems: an introduction", EURO
Journal on Transportation and Logistics, Vol. 1, No. 4, pp. 307-326, 2012.

[5] Y. Adulyasak, J-F. Cordeau, and R. Jans, "The production routing problem: A review of formulations and solution algorithms", Computers and Operations Research, Vol. 55, pp. 141-152, 2015.

[6] P. Chandra and M.L. Fisher, "Coordination of production and distribution planning", European Journal of Operational Research, Vol. 72, No. 3, pp. 503-517, 1994.

[7] L. Lei, S. Lei, A. Ruszczynski, and S. Park, "On the integrated production, inventory, and distribution routing problem", IIE Transactions, Vol. 38, No. 11, pp. 955-970, 2006.

[8] M. Boudia, M. A. O. Louly, and C. Prins, "A reactive GRASP and path relinking for a combined production-distribution problem", Computers and Operations Research, Vol. 34, pp. 3402-3419, 2007.

[9] M. Boudia, M. A. O. Louly, and C. Prins, "Fast heuristics for a combined production planning and vehicle routing problem", Production Planning and Control, Vol. 19, No. 2, pp. 85-96, 2008.

[10] M. Boudia and C. Prins, "A memetic algorithm with dynamic population management for an integrated production-distribution problem", European Journal of Operational Research, Vol. 195, No. 3, pp. 703-715, 2009.

[11]J.F. Bard and N. Nananukul, "The integrated production-inventory-distribution-routing problem", Journal of Scheduling, Vol. 12, No. 3, pp. 257-80, 2009.

[12] J. F. Bard and N. Nananukul, "Heuristics for a multi-period inventory routing problem with production decisions", Computers and Industrial Engineering, Vol. 57, No. 3, pp. 713-23, 2009.

[13]J. F. Bard and N. Nananukul, “A branch-and-price algorithm for an integrated production and inventory routing problem", Computers and 
Operations Research, Vol. 37, No. 12, pp. 22022217, 2010.

[14] A. Al-Fazary, A. Eltawil, and M. N. Fors, "A proposed model for integrated productiondistribution planning", Proceedings of the 19th International Conference on Management of Technology (IAMOT), Cairo, Egypt, 2010.

[15]C. Archetti, L. Bertazzi, G. Paletta and M.G. Speranza, "Analysis of the maximum level policy in a production-distribution system", Computers and Operations Research, Vol. 38, No. 12, pp. 1731-1746, 2011.

[16] V. A. Armentano, A.L. Shiguemoto, and A. Lokketangen, "Tabu search with path relinking for an integrated production-distribution problem", Computers and Operations Research, Vol. 38, No. 8, pp. 1199-1209, 2011.

[17] K. Piewthongngam, S. Pathumnakul, and S. Homkhampad, "An interactive approach to optimize production-distribution planning for an integrated feed swine company", International Journal of Production Economics, Vol. 142, No. 2, pp. 290-301, 2013

[18]N. Absi, C. Archetti, S. Dauzère-Pérès, and D. Feillet, "A two-phase iterative heuristic approach for the production routing problem",
Transportation Science, forthcoming, published online: July 2014.

[19]Y. Adulyasak, J-F. Cordeau, and R. Jans, "Formulations and branch and cut algorithms for multi-vehicle production and inventory routing problems", INFORMS Journal on Computing, Vol. 26, No. 1, pp. 103-20, 2014.

[20]S.M. Seyedhosseini and S.M. Ghoreyshi, "An integrated model for production and distribution planning of perishable products with inventory and routing considerations", Mathematical Problems in Engineering, Vol. 2014, pp. 1-10, 2014.

[21]G.K. Janssens, A. Caris, and K. Ramaekers, "Time Petri nets as an evaluation tool for handling travel time uncertainty in vehicle routing solutions", Expert Systems with Applications, Vol. 36, pp. 5987-5991, 2009.

[22]T. Bektas, G. Laporte, "The Pollution-Routing Problem," Transportation Research Part B, Vol. 45, pp 1232-1250, 2011

[23]Lamis E. Amer, Amr B. Eltawil, Analysis of Quantitative Models of Horizontal Collaboration in Supply Chain Network Design, Towards "Green Collaborative" Strategies, Proceedings of the Industrial Engineering and Operations Management (IEOM), Dubai, UAE, 2015. 